\title{
The semigroup generated by the similarity class of a singular matrix
}

\author{
Clément de Seguins Pazzis*†
}

November 21, 2018

\begin{abstract}
Let $A$ be a singular matrix of $\mathrm{M}_{n}(\mathbb{K})$, where $\mathbb{K}$ is an arbitrary field. Using canonical forms, we give a new proof that the sub-semigroup of $\left(\mathrm{M}_{n}(\mathbb{K}), \times\right)$ generated by the similarity class of $A$ is the set of matrices of $\mathrm{M}_{n}(\mathbb{K})$ with a rank lesser than or equal to that of $A$.
\end{abstract}

\section{AMS Classification: Primary 15A30. Secondary 15A23.}

Keywords: semigroup, similarity class, companion matrices, rational canonical form, Jordan canonical form.

\section{Introduction}

Let $\mathbb{K}$ be an arbitrary (commutative) field, and denote by $\mathrm{M}_{n}(\mathbb{K})$ the algebra of square matrices with $n$ rows and entries in $\mathbb{K}$, and by $\mathrm{GL}_{n}(\mathbb{K})$ its group of invertible elements.

Our starting point is the famous theorem of J.A. Erdos [7, which states that every singular matrix of $\mathrm{M}_{n}(\mathbb{K})$ is a product of idempotent ones (for alternative proofs, see [1], 3], 6] and [13]; for an extension to principal ideal domains, see [5]). Erdos's theorem and Howie's set-theoretic counterpart [12] have had a significant impact in inspiring the definition of independence algebras (see [10] and [8]).

\footnotetext{
*Lycée Privé Sainte-Geneviève, 2, rue de l'École des Postes, 78029 Versailles Cedex, FRANCE.

${ }^{\dagger}$ e-mail: dsp.prof@gmail.com
} 
A trivial consequence of Erdos's theorem is that every singular matrix of $\mathrm{M}_{n}(\mathbb{K})$ is a product of rank $n-1$ idempotent matrices. Since the rank $n-1$ idempotent matrices form a single similarity class, a natural extension of the question is to determine the sub-semigroup of $\left(\mathrm{M}_{n}(\mathbb{K}), \times\right)$ generated by the similarity class of a given singular matrix $A$. It is straightforward that this semigroup is included in the semigroup consisting of the matrices $M \in \mathrm{M}_{n}(\mathbb{K})$ such that $\operatorname{rk} M \leq \operatorname{rk} A$. Remarkably enough, the converse also holds, as was originally proven independently by Araújo and Silva [2] and Grunenfelder, Omladič, Radjavi and Sourour [11]. The purpose of this note is to give an efficient and relatively elementary proof of that statement which works regardlessly of the ground field (unlike Araújo and Silva's proof) and does not rely on prior results about semigroups generated by the similarity class of an invertible matrix (unlike Grunenfelder et al's proof). Let us first restate the main theorem:

Theorem 1. Let $A$ be a rank $p$ singular matrix of $\mathrm{M}_{n}(\mathbb{K})$. Then the semi-group generated by the similarity class of $A$ is

$$
S_{p}:=\left\{M \in \mathrm{M}_{n}(\mathbb{K}): \operatorname{rk} M \leq p\right\} .
$$

Notice the straightforward corollary:

Corollary 2. The sub-semigroups of $\mathrm{M}_{n}(\mathbb{K})$ which are invariant under conjugation and contain only singular matrices are the $S_{k}$ 's for $k \in\{0, \ldots, n-1\}$.

Before explaining our proof, we need to recall a few standard notations: given a monic polynomial $P=t^{p}-\sum_{k=0}^{p-1} a_{k} t^{k}$ (with indeterminate $t$ ), we denote its companion matrix by

$$
C(P):=\left[\begin{array}{ccccc}
0 & & & 0 & a_{0} \\
1 & 0 & & & a_{1} \\
0 & \ddots & \ddots & & \vdots \\
\vdots & & & 0 & a_{p-2} \\
0 & & & 1 & a_{p-1}
\end{array}\right] \in \mathrm{M}_{p}(\mathbb{K})
$$


We also introduce the (nilpotent) Jordan matrix:

$$
J_{p}:=C\left(t^{p}\right)^{T}=\left[\begin{array}{ccccc}
0 & 1 & 0 & \cdots & 0 \\
0 & 0 & 1 & & \vdots \\
& & \ddots & \ddots & 0 \\
\vdots & & & 0 & 1 \\
0 & \cdots & & & 0
\end{array}\right] \in \mathrm{M}_{p}(\mathbb{K}) .
$$

Similarity of two matrices $A$ and $B$ will be denoted by $A \sim B$, and the blockdiagonal matrix with diagonal blocks $A_{1}, \ldots, A_{d}$ will be denoted by $\operatorname{Diag}\left(A_{1}, \ldots, A_{d}\right)$.

\section{Proof of the theorem}

Let $A \in \mathrm{M}_{n}(\mathbb{K})$ with rank $p<n$ and denote by $S$ the semigroup generated by the similarity class of $A$. Note that $S$ is invariant under conjugation and $S \subset S_{p}$. Our goal is to show that $S=S_{p}$. There are three steps:

Step 1. $S$ contains a rank $p$ matrix $M$ such that $\operatorname{Ker} M \oplus \operatorname{Im} M=\mathbb{K}^{n}$ (i.e. for which 0 is a semi-simple eigenvalue).

Step 2. $S$ contains a rank $p$ idempotent matrix.

Step 3. $S$ contains every rank $p$ matrix $M$ for which 0 is a semi-simple eigenvalue.

Step 4. $S$ contains every rank $p$ matrix of $\mathrm{M}_{n}(\mathbb{K})$.

Step 5. $S$ contains every matrix $N$ of $\mathrm{M}_{n}(\mathbb{K})$ such that $\mathrm{rk} N<p$.

\subsection{Proof of Step 1}

Using the Fitting decomposition 11 and the Jordan canonical form 2 for the nilpotent part in this decomposition, one finds a non-singular matrix $Q \in \mathrm{GL}_{q}(\mathbb{K})$ and positive integers $i_{1}, \ldots, i_{N}$ such that

$$
A \sim \operatorname{Diag}\left(Q, J_{i_{1}}, \ldots, J_{i_{N}}\right) .
$$

\footnotetext{
${ }^{1}$ See for example 4 .

${ }^{2}$ See for example 9
} 
However $J_{k} \sim J_{k}^{T}$ for every positive integer $k$ (simply conjugate $J_{k}$ with a wellchosen permutation matrix) hence $S$ contains the product

$B=\operatorname{Diag}\left(Q, J_{i_{1}}, \ldots, J_{i_{N}}\right) \operatorname{Diag}\left(Q, J_{i_{1}}^{T}, \ldots, J_{i_{N}}^{T}\right)=\operatorname{Diag}\left(Q^{2}, I_{i_{1}-1}, 0, I_{i_{2}-1}, 0 \ldots, I_{i_{N}-1}, 0\right)$.

Since $Q^{2}$ is non-singular, one has $\operatorname{rk} B=n-N=\operatorname{rk} A$ and obviously $\operatorname{Im} B \oplus$ $\operatorname{Ker} B=\mathbb{K}^{n}$.

\section{$2.2 \quad$ Proof of Step 2}

Amongst the rank $p$ matrices $M \in S$ satisfying $\operatorname{Im} M \oplus \operatorname{Ker} M=E$, we choose one for which $\operatorname{rk}\left(M-I_{n}\right)$ is minimal. We claim that $M$ is idempotent: to prove this, it suffices to show that $\operatorname{dim} \operatorname{Ker}\left(M-I_{n}\right)=p$. We perform a reductio ad absurdum and assume $\operatorname{dim} \operatorname{Ker}\left(M-I_{n}\right)<p$. We obviously lose no generality assuming that

$$
M=\operatorname{Diag}\left(N, 0_{n-p}\right) \quad \text { for some } N \in \mathrm{GL}_{p}(\mathbb{K}) .
$$

In the rational canonical form of $N$, one of the elementary polynomials is not $t-1$ : we choose such a polynomial $P(t)$, denote by $d$ its degree, and we may then find a non-singular matrix $Q$ such that

$$
N \sim \operatorname{Diag}(Q, C(P(t)))
$$

Notice that $P(0) \neq 0$, hence the matrix $N^{\prime}:=\operatorname{Diag}(C(P(t)), 0)$ of $\mathrm{M}_{d+1}(\mathbb{K})$ has $t P(t)$ as minimal polynomial and is therefore similar to $C(t P(t))$. We thus lose no generality assuming that

$M=\operatorname{Diag}\left(Q, C(t P(t)), 0_{n-p-1}\right) \quad$ (where the last diagonal block may be empty).

Now, set

$$
K=\left[\begin{array}{ccc}
(0) & & 1 \\
& . & \\
1 & & (0)
\end{array}\right]=\left(\delta_{i+j, d+2}\right)_{1 \leq i, j \leq d+1} .
$$

A straightforward computation yields:

$$
K C(t P(t)) K^{-1} C(t P(t))=\left[\begin{array}{cc}
I_{d} & C \\
0 & 0
\end{array}\right]
$$


for some column-matrix $C \in \mathrm{M}_{d+1,1}(\mathbb{K})$, hence

$$
K C(t P(t)) K^{-1} C(t P(t)) \sim\left[\begin{array}{cc}
I_{d} & 0 \\
0 & 0
\end{array}\right] \text {. }
$$

It follows that $S$ contains the product

$M^{\prime}:=\operatorname{Diag}\left(Q, K C(t P(t)) K^{-1}, 0_{n-p-1}\right) \times \operatorname{Diag}\left(Q, C(t P(t)), 0_{n-p-1}\right) \sim \operatorname{Diag}\left(Q^{2}, I_{d}, 0_{n-p}\right)$,

for which 0 is a semi-simple eigenvalue. The assumptions on $P(t)$ show that $\operatorname{dim} \operatorname{Ker}\left(C(P(t))-I_{d}\right)<d$, whereas $\operatorname{rk}\left(Q^{2}-I_{n}\right) \leq \operatorname{rk}\left(Q-I_{n}\right)$ : therefore $\operatorname{rk}\left(M^{\prime}-\right.$ $\left.I_{n}\right)<\operatorname{rk}\left(M-I_{n}\right)$, which contradicts the definition of $M$ and completes Step 2.

\subsection{Proof of Step 3}

Let $B$ be a rank $p$ matrix of $\mathrm{M}_{n}(\mathbb{K})$ for which 0 is a semi-simple eigenvalue. Hence

$$
B \sim \operatorname{Diag}\left(Q, 0_{n-p}\right) \quad \text { for some } Q \in \mathrm{GL}_{p}(\mathbb{K}) .
$$

By Erdos's theorem [7], the matrix $B^{\prime}:=\operatorname{Diag}(Q, 0) \in \mathrm{M}_{p+1}(\mathbb{K})$ may be decomposed as $B^{\prime}=P_{1} \cdots P_{N}$ where the $P_{k}$ 's are rank $p$ idempotent matrices. Then, for each $k \in\{1, \ldots, N\}$, the matrix $Q_{k}:=\operatorname{Diag}\left(P_{k}, 0_{n-p-1}\right)$ is a rank $p$ idempotent, and it therefore belongs to $S$ by the previous step. However

$$
Q_{1} \cdots Q_{N}=\operatorname{Diag}\left(Q, 0_{n-p}\right) \sim B
$$

hence $B \in S$, which completes the present step.

\subsection{Proof of Step 4}

Let $B \in \mathrm{M}_{n}(\mathbb{K})$ be a rank $p$ matrix. Set $d:=\operatorname{dim}(\operatorname{Ker} B \cap \operatorname{Im} B)$. If $d=0$, then we know that $B \in S$. Assume that $d \geq 1$. Using once more the Fitting decomposition and the Jordan canonical form for the nilpotent part of it, we find an integer $q$, a matrix $P \in \mathrm{GL}_{q}(\mathbb{K})$, and integers $i_{1}, \ldots, i_{d}$ all greater than 1 such that

$$
B \sim \operatorname{Diag}\left(P, J_{i_{1}}, \ldots, J_{i_{d}}, 0_{n-p-d}\right) \sim \operatorname{Diag}\left(P, J_{i_{1}}^{T}, \ldots, J_{i_{d}}^{T}, 0_{n-p-d}\right) .
$$

For $k \geq 2$, set

$$
C_{k}:=C\left(t^{k}-t\right) \sim \operatorname{Diag}\left(C\left(t^{k-1}-1\right), 0\right) \quad \text { and } \quad C_{k}^{\prime}:=\operatorname{Diag}\left(I_{k-1}, 0\right) \in \mathrm{M}_{k}(\mathbb{K})
$$


and notice that these are rank $k-1$ matrices with 0 as semi-simple eigenvalue. A straightforward computation shows that

$$
\forall k \geq 2, J_{k}^{T}=C_{k} C_{k}^{\prime}
$$

hence

$\operatorname{Diag}\left(P, J_{i_{1}}^{T}, \ldots, J_{i_{d}}^{T}, 0_{n-p-d}\right)=\operatorname{Diag}\left(P, C_{i_{1}}, \ldots, C_{i_{d}}, 0_{n-p-d}\right) \times \operatorname{Diag}\left(I_{q}, C_{i_{1}}^{\prime}, \ldots, C_{i_{d}}^{\prime}, 0_{n-p-d}\right)$

and the matrices $\operatorname{Diag}\left(P, C_{i_{1}}, \ldots, C_{i_{d}}, 0_{n-p-d}\right)$ and $\operatorname{Diag}\left(I_{q}, C_{i_{1}}^{\prime}, \ldots, C_{i_{d}}^{\prime}, 0_{n-p-d}\right)$ have rank $p$ with 0 as semi-simple eigenvalue. It follows from Step 3 that both belong to $S$, and hence $B \in S$.

\subsection{Proof of Step 5}

The line of reasoning here is classical but we reproduce it for the sake of completeness. Let $B \in \mathrm{M}_{n}(\mathbb{K})$ with rank $r<p$. Then there are non-singular matrices $Q$ and $Q^{\prime}$ of $\mathrm{M}_{n}(\mathbb{K})$ such that

$$
B=Q \operatorname{Diag}\left(I_{r}, 0_{n-r}\right) Q^{\prime} .
$$

For $k \in\{r+1, \ldots, p+1\}$, set $D_{k}:=\operatorname{Diag}\left(I_{k-1}, 0, I_{p+1-k}, 0_{n-p-1}\right)$ and notice that $D_{r+1} \cdots D_{p+1}=\operatorname{Diag}\left(I_{r}, 0_{n-r}\right)$, hence

$$
B=\left(Q D_{r+1}\right) D_{r+2} \cdots D_{p}\left(D_{p+1} Q^{\prime}\right)
$$

is a product of rank $p$ matrices and therefore belongs to $S$ by Step 4 . This completes Step 5, which finishes our proof of Theorem 1 .

\section{$3 \quad$ Suggested problems}

We would like to suggest two problems on the issue of semigroups of matrices generated by a similarity class:

(1) Describe the semigroup generated by the similarity class of an invertible matrix with entries in an infinite field. The case where the determinant of the matrix is a root of unity has been solved in [11] but the solution of the general case is not known yet.

(2) Find a truly elementary proof of Theorem 1, analogous to the one in [1]. 


\section{Acknowledgements}

The author would like to thank the referee for his many thoughtful suggestions.

\section{References}

[1] J. Arajo, J.D. Mitchell, An elementary proof that every singular matrix is a product of idempotent matrices, Amer. Math. Monthly 112 (2005) 641-645.

[2] J. Araújo, F.C. Silva, Semigroups of linear endomorphisms closed under conjugation, Comm. Algebra 28 (8) (2000) 3679-3689.

[3] C.S. Ballantine, Products of idempotent matrices, Linear Algebra Appl. 19 (1) (1978) 81-86.

[4] D. Benson, Representations and Cohomology I, Cambridge Studies in Advanced Mathematics 301991.

[5] K.P.S. Bhaskara Rao, Products of idempotent matrices over integral domains, Linear Algebra Appl. 430 (2009) 2690-2695.

[6] D.Z. Djokovic, Note on a theorem on singular matrices, Linear Algebra Appl. 11 (1968) 283-284.

[7] J.A. Erdos, On products of idempotent matrices, Glasgow Math. J. 8 (1967) 118-122.

[8] J. Fountain, A. Lewin, Product of idempotent endomorphisms of an independence algebra of finite rank, Proc. Edinburgh Math. Soc. (2) 35 (3) (1992) 493-500.

[9] F.R. Gantmacher, Matrix Theory, Vol. 1 New York: Chelsea, 1977.

[10] V. Gould, Independence algebras, Algebra Universalis 33 (1995) 294-318.

[11] L. Grunenfelder, M. Omladič, H. Radjavi, A. Sourour, Semigroups generated by similarity orbits, Semigroup Forum 62 (3) (2001) 460-472.

[12] J.D. Howie, The Subsemigroup Generated By the Idempotents of a Full Transformation Semigroup, J. London Math. Soc. (ser. 1) 41 (1966) 707716. 
[13] J.B. Kim, Idempotent generated Rees matrix semigroups, Kyungpook Math. J. 10 (1970) 7-13. 